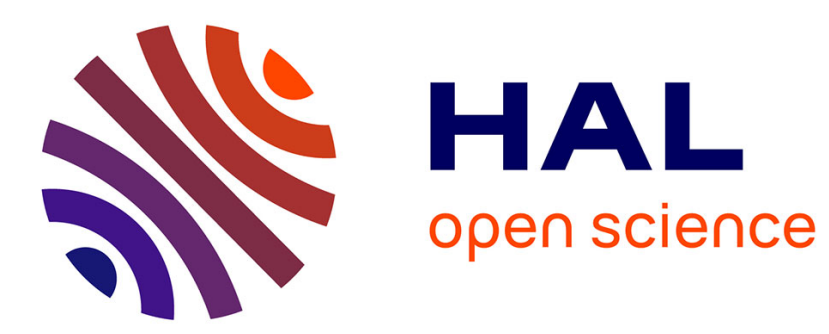

\title{
The Discrete Relations Between Fields and Potentials with High Order Whitney Forms
}

Ana Alonso Rodríguez, Francesca Rapetti

\section{To cite this version:}

Ana Alonso Rodríguez, Francesca Rapetti. The Discrete Relations Between Fields and Potentials with High Order Whitney Forms. Numerical Mathematics and Advanced Applications ENUMATH 2017, Springer, pp.259-267, 2019, 978-3-319-96415-7. 10.1007/978-3-319-96415-7_22 . hal-02411197

\section{HAL Id: hal-02411197 \\ https://hal.science/hal-02411197}

Submitted on 16 Dec 2019

HAL is a multi-disciplinary open access archive for the deposit and dissemination of scientific research documents, whether they are published or not. The documents may come from teaching and research institutions in France or abroad, or from public or private research centers.
L'archive ouverte pluridisciplinaire HAL, est destinée au dépôt et à la diffusion de documents scientifiques de niveau recherche, publiés ou non, émanant des établissements d'enseignement et de recherche français ou étrangers, des laboratoires publics ou privés. 


\title{
The discrete relations between fields and potentials with high order Whitney forms
}

\author{
Ana Alonso Rodríguez and Francesca Rapetti \\ 1 Dip. di Matematica, Università degli Studi di Trento, Povo, Trento, Italy \\ ana.alonso@unitn.it \\ 2 Dep. de Mathématiques J.-A. Dieudonné, Univ. Côte d'Azur, Nice, France \\ frapetti@unice.fr
}

\begin{abstract}
When using the lower order Whitney elements on a simplicial complex, the matrices describing the external derivative, namely, the differential operators gradient, curl and divergence, are the incidence matrices between edges and vertices, faces and edges, tetrahedra and faces. For higher order Whitney elements, if one adopts degrees of freedom based on moments, the entries of these matrices are still equal to 0,1 or -1 but they are no more incidence matrices. If one uses instead the "weights of the field on small simplices" as alternative degrees of freedom, the matrices representative of the external derivative are incidence matrices for any polynomial degree. ${ }^{1}$
\end{abstract}

\section{Introduction}

Together with the list of nodes and of their positions, the mesh data structure also contains incidence matrices (with entries 0,1 or -1 ) saying which node belongs to which edge, which edge bounds which face, etc., and there is a notion of (inner) orientation of the simplices to consider. In short, an edge, a face, is not only a two-node $\left[n_{i}, n_{j}\right]$, three-node $\left[n_{i}, n_{j}, n_{l}\right]$, subset of the set of the mesh nodes, but such a set plus an orientation of the simplex it subtends that is defined by the order of its vertices $\left(e . g,\left[n_{i}, n_{j}\right]\right.$ is the edge oriented from $n_{i}$ to $n_{j}$ ). These matrices, besides containing all the information about the topology of the domain, for the lowest approximation polynomial degree, they help connecting the degrees of freedom (dofs) describing the potentials to those describing the fields. As an example, the relation $\mathbf{E}=-\operatorname{grad} V$ at the continuous level becomes $\mathbf{e}=-G \mathbf{v}$ where $G$ coincides with the node-toedge incidence matrix and $\mathbf{e}$ (resp. $\mathbf{v}$ ) is the vector of edge circulations (resp. values at nodes) of the electric field $\mathbf{E}$ (resp. the scalar electric potential $V$ ), when nodal (resp. edge) finite elements are used to approximate $V$ (resp. E). As pointed out in [4], nodal and edge finite element approximations, that belong to the lowest order reconstructions by the finite element families introduced in [6] for $\mathbb{R}^{3}$ and in [9] for $\mathbb{R}^{2}$, are Whitney differential forms [10]. We wish to investigate the block-structure of the incidence matrices, when

\footnotetext{
1 The first author thanks the Laboratoire de Mathématiques J.A. Dieudonné, Uni-
} versité Côte d'Azur, Nice, France, where this work started. 
fields and potentials are approximated by high order Whitney forms [3,8], with dofs given either by the well-known moments [6] or by the more recent weights on the small simplices $[7,8]$. We do not have in mind a particular differential equation to solve but we work on the algebraic equivalent of the field/potential relation when they both are approximated by forms of higher polynomial degree. We will see that the involved matrices present a structure by blocks, each block multiplying the dofs of the potential associated to a geometrical dimension. In the following we adopt the notation of the finite element exterior calculus, see e.g. [2].

Let $\mathcal{P}_{r}\left(\mathbb{R}^{d}\right)$ and $\mathcal{H}_{r}\left(\mathbb{R}^{d}\right)$ be, respectively, the space of polynomials in $n$ variables of degree at most $r$ and the space of homogenous polynomials in $n$ variables of degree equal to $r$. Then, $\mathcal{P}_{r} \Lambda^{k}\left(\mathbb{R}^{d}\right)=\mathcal{P}_{r} \Lambda^{k}$ and $\mathcal{H}_{r} \Lambda^{k}\left(\mathbb{R}^{d}\right)=$ $\mathcal{H}_{r} \Lambda^{k}$ denote the corresponding spaces of polynomial differential forms. The space of trimmed polynomial differential forms $\mathcal{P}_{r}^{-} \Lambda^{k}$ can be defined by involving the Koszul operator, $\kappa: \Lambda^{k+1} \rightarrow \Lambda^{k}$ being $\Lambda^{k}$ the space of $k$ differential forms in $\mathbb{R}^{d}$ (see, e.g. [1]), that satisfies

$$
\kappa(u \wedge \eta)=(\kappa u) \wedge \eta+(-1)^{k} u \wedge(\kappa \eta), \quad u \in \Lambda^{k}, \quad \eta \in \Lambda^{2} .
$$

Moreover, $\kappa(f u)=f \kappa u$ if $f$ is a function and $\kappa\left(\mathrm{d} x_{i}\right)=x_{i}$. These properties fully determine $\kappa$ and yield $\kappa\left(\mathrm{d} x_{i} \wedge \mathrm{d} x_{j}\right)=x_{i} \mathrm{~d} x_{j}-x_{j} \mathrm{~d} x_{i}, \kappa\left(\mathrm{d} x_{i} \wedge \mathrm{d} x_{j} \wedge \mathrm{d} x_{k}\right)=$ $x_{i} \mathrm{~d} x_{j} \wedge \mathrm{d} x_{k}-x_{j} \mathrm{~d} x_{i} \wedge \mathrm{d} x_{k}+x_{k} \mathrm{~d} x_{i} \wedge \mathrm{d} x_{j}$. In particular $\kappa: \mathcal{H}_{r} \Lambda^{k+1} \rightarrow \mathcal{H}_{r+1} \Lambda^{k}$ and, for $d=3, \kappa u$ is equal to $\mathbf{x} \cdot \mathbf{u}(k=0), \mathbf{x} \times \mathbf{u}(k=1), \mathbf{x} u(k=2)$, and 0 $(k=3)$, respectively. The space $\mathcal{P}_{r}^{-} \Lambda^{k}$ is intermediate between $\mathcal{P}_{r-1} \Lambda^{k}$ and $\mathcal{P}_{r} \Lambda^{k}$ as follows:

$$
\mathcal{P}_{r}^{-} \Lambda^{k}=\mathcal{P}_{r-1} \Lambda^{k}+\kappa \mathcal{H}_{r-1} \Lambda^{k+1}=\left\{u \in \mathcal{P}_{r} \Lambda^{k}: \kappa u \in \mathcal{P}_{r} \Lambda^{k-1}\right\} .
$$

Let $\Omega$ be a bounded polyhedral domain of $\mathbb{R}^{d}$ and $\mathcal{T}$ a simplicial mesh of $\Omega$. The spaces of finite element differential forms with respect to the triangulation $\mathcal{T}$ are denoted $\mathcal{P}_{r}^{-} \Lambda^{k}(\mathcal{T})$. By $H \Lambda^{k}(\Omega)$ we denote the Sobolev space $H^{1}(\Omega)$ if $k=0, H(\operatorname{curl} ; \Omega)$ if $k=1, H(\operatorname{div} ; \Omega)$ if $k=2$ and the space $L^{2}(\Omega)$ if $k=3$. Then we define

$$
\mathcal{P}_{r}^{-} \Lambda^{k}(\mathcal{T})=\left\{u \in H \Lambda^{k}(\Omega): u_{\mid T} \in \mathcal{P}_{r}^{-} \Lambda^{k} \text { for all } T \in \mathcal{T}\right\} .
$$

It is well known (see, for instance, [2]) that these finite element spaces are that of the Lagrange finite elements of degree $r$ if $k=0$, the first family of Nédélec finite elements of order $r$ conforming in $H(\operatorname{curl} ; \Omega)$ if $k=1$, the first family of Nédélec finite elements of order $r$ conforming in $H(\operatorname{div} ; \Omega)$ if $k=2$, and discontinuous elements of degree $\leq r-1$ if $k=3$.

For $0 \leq j \leq d$, let $\Delta_{j}(T)$ be the set of all $j$-dimensional subsimplices of the simplex $T$ and $\Delta_{j}(\mathcal{T})$ be the set of all $j$-dimensional subsimplices of the mesh $\mathcal{T}$. Let us denote by $\Lambda^{k}(T)$ the space of all smooth differential $k$-forms on a tetrahedron $T$. The trace operator $\operatorname{Tr}_{S}: \Lambda^{k}(T) \rightarrow \Lambda^{k}(S)$, for $S \in \Delta_{j}(T)$ 
with $k \leq j<3$, is the map defined in the following way

$$
\begin{aligned}
& \text { for } j=2(S=f) \quad \text { for } j=1(S=e) \quad \text { for } j=0\left(S=n_{i}\right) \\
& \operatorname{Tr}_{f} u=\left\{\begin{array}{l}
u_{\mid f} \quad \text { if } k=0, \\
\mathbf{n}_{f} \times \mathbf{u} \text { if } k=1, \\
\mathbf{u} \cdot \mathbf{n}_{f} \text { if } k=2 ;
\end{array} \quad \operatorname{Tr}_{e} u=\left\{\begin{array}{ll}
u_{\mid e} & \text { if } k=0, \\
\mathbf{u} \cdot \boldsymbol{\tau}_{e} & \text { if } k=1 ;
\end{array} \quad \operatorname{Tr}_{n_{i}} u=u\left(n_{i}\right) \text { if } k=0 .\right.\right.
\end{aligned}
$$

where $\mathbf{n}_{f}=\frac{\left(\mathbf{v}_{j}-\mathbf{v}_{i}\right) \times\left(\mathbf{v}_{k}-\mathbf{v}_{i}\right)}{\left|\left(\mathbf{v}_{j}-\mathbf{v}_{i}\right) \times\left(\mathbf{v}_{k}-\mathbf{v}_{i}\right)\right|}$, for $f=\left[n_{i}, n_{j}, n_{k}\right], \boldsymbol{\tau}_{e}=\frac{\mathbf{v}_{j}-\mathbf{v}_{i}}{\left|\mathbf{v}_{j}-\mathbf{v}_{i}\right|}$, if $e=\left[n_{i}, n_{j}\right]$, with $\mathbf{v}_{i}$ the Cartesian coordinates of the node $n_{i}$.

In the finite element exterior calculus, we adopt spaces of differential forms $u$ which are piecewise smooth, usually polynomials, with respect to $\mathcal{T}$, i.e., the restriction $u_{\mid T}$ is smooth for each $T \in \mathcal{T}$. Then, for $f \in \Delta_{j}(\mathcal{T})$ with $j \geq k$, the form $\operatorname{Tr}_{f} u$ can be multi-valued, that is, we can assign a value for each $T \in \mathcal{T}$ containing $f$ by restricting $u$ to $T$ and then taking the trace on $f$. If all such traces coincide, we say that $\operatorname{Tr}_{f} u$ is single-valued. It is well-known that if $\operatorname{Tr}_{f} u$ is single-valued for all $f \in \Delta_{j}(\mathcal{T}), k \leq j \leq d-1$, then $u \in H \Lambda^{k}(\Omega)$

\section{Moments and potentials}

The construction of dofs based on moments for the space $\mathcal{P}_{r}^{-} \Lambda^{k}(T)$ requires the use of $\mathcal{P}_{r} \Lambda^{k}(f)$, for certain $f$ subsimplices of $T$. Here, $\mathcal{P}_{r} \Lambda^{k}(f)$ is the space of forms obtained by restricting those of $\mathcal{P}_{r} \Lambda^{k}\left(\mathbb{R}^{s}\right)$ to $f$ being $s=\operatorname{dim}(f) \leq d$.

Definition 1. The moments of a polynomial $k$-form $u \in \mathcal{P}_{r}^{-} \Lambda^{k}(T)$ with $0 \leq k \leq n$ and $r \geq 1$ are the scalar quantities

$$
\int_{f} \operatorname{Tr}_{f} u \wedge \eta, \quad \eta \in \mathcal{P}_{r+k-s-1} \Lambda^{s-k}(f),
$$

for each $f \in \Delta_{s}(T)$ and $k \leq s=\operatorname{dim}(f) \leq d$.

The moments of a $k$-form $u$ are associated to subsimplices of $T$ of dimension equal to or greater than $k$. If $r+k-s-1<0$ in (1), there are no moments associated to subsimplices of dimension $s$. For $r=1$, the moments are associated only to $k$-subsimplices of $T$. The wedge product $\wedge$ appearing in (1) extends the notion of exterior product to forms. It holds that $u^{(k)} \wedge v^{(\ell)}=(-1)^{k \ell} v^{(\ell)} \wedge u^{(k)}$, where $u^{(k)}$ stands for the $k$-form $u$. If $k+\ell>d$ it is zero. In details, for $d=2,3$, we have $u^{(0)} \wedge v^{(k)}=(u v)^{(k)}$ for $k \leq d$, $u^{(1)} \wedge v^{(1)}=(u \times v)^{(2)}$, and, for $d=3, u^{(1)} \wedge v^{(2)}=u^{(2)} \wedge v^{(1)}=(u \cdot v)^{(3)}$.

When considering moments the main tool for the identification of the matrix of the exterior derivative operator is the integration by parts formula

$$
\int_{f} \operatorname{Tr}_{f} \mathrm{~d} u \wedge \eta=\int_{\partial f} \operatorname{Tr}_{\partial f}\left(\operatorname{Tr}_{f} u \wedge \eta\right)+(-1)^{k-1} \int_{f} \operatorname{Tr}_{f} u \wedge \mathrm{d} \eta, \quad u \in \Lambda^{k}(T) .
$$

Another important tool is the choice of a basis of $\mathcal{P}_{r+k-s-1} \Lambda^{s-k}(f)$ to represent $\eta$. To this purpose, we introduce the multi-index $\boldsymbol{\alpha}=\left(\alpha_{0}, \ldots, \alpha_{s}\right)$ 
of $s+1$ integers $\alpha_{i} \geq 0$ and weight $|\boldsymbol{\alpha}|=\sum_{i=1}^{s} \alpha_{i}$. The set of multiindices $\boldsymbol{\alpha}$ with $s+1$ components and weight $r$ is denoted $\mathcal{I}(s+1, r)$. Any (oriented) 3 -simplex $T=\left[n_{i} . n_{j}, n_{l}, n_{p}\right]$ is associated to an increasing map $\sigma_{T}^{0}:\{0123\} \rightarrow\left\{12 \ldots N_{v}\right\}$ and $T=\left[n_{\sigma_{T}^{0}(0)}, \ldots, n_{\sigma_{T}^{0}(3)}\right]$. Any (oriented) s-simplex $S \in \Delta_{s}(T)$ is associated to an increasing map $\sigma_{S}^{1}:\{01 \ldots s\} \rightarrow$ $\{0123\}$ such that if $\sigma_{S}=\sigma_{S}^{1} \circ \sigma_{T}^{0}$ then $S=\left[n_{\sigma_{f}(0)}, \ldots, n_{\sigma_{f}(s)}\right]$. For each $S \in \Delta_{s}(T)$ and $\boldsymbol{\alpha} \in \mathcal{I}(s+1, r)$ we set $\lambda^{\boldsymbol{\alpha}}=\lambda_{\sigma_{S}(0)}^{\alpha_{0}} \ldots \lambda_{\sigma_{S}(s)}^{\alpha_{s}}$. Then

$$
\begin{aligned}
& \mathcal{P}_{r^{\prime}} \Lambda^{0}(S)=\left\{\lambda^{\alpha}: \boldsymbol{\alpha} \in \mathcal{I}\left(s+1, r^{\prime}\right)\right\}, \\
& \mathcal{P}_{r^{\prime}} \Lambda^{1}(S)=\left\{\lambda^{\alpha} \mathrm{d} \lambda_{i}: \boldsymbol{\alpha} \in \mathcal{I}\left(s+1, r^{\prime}\right), i \in\left\{\sigma_{S}(1), \ldots \sigma_{S}(s)\right\}\right\} \\
& \mathcal{P}_{r^{\prime}} \Lambda^{2}(S)=\left\{\lambda^{\boldsymbol{\alpha}} \mathrm{d} \lambda_{i} \wedge \mathrm{d} \lambda_{j}: \boldsymbol{\alpha} \in \mathcal{I}\left(s+1, r^{\prime}\right), i, j \in\left\{\sigma_{S}(1), \ldots \sigma_{S}(s)\right\}, i<j\right\} \\
& \mathcal{P}_{r^{\prime}} \Lambda^{3}(S)=\left\{\lambda^{\boldsymbol{\alpha}} \mathrm{d} \lambda_{i} \wedge \mathrm{d} \lambda_{j} \wedge \mathrm{d} \lambda_{\ell}: \boldsymbol{\alpha} \in \mathcal{I}\left(s+1, r^{\prime}\right), i, j, \ell \in\left\{\sigma_{S}(1), \ldots \sigma_{S}(s)\right\}\right. \\
& i<j<\ell\}
\end{aligned}
$$

We now detail (2) in the case of the divergence, the curl and the gradient operators in order to better understand the structure of the matrices representing these differential operators at the discrete level. An example of construction of this matrix on one element $T=[0,1,2,3]$ is given for $r=2$. The missing blocks in the matrices of the Examples are full of zeros.

(i) The discrete relation between the moments of the discontinuous piecewise polynomial function $\operatorname{div} \mathbf{u}$ and those of the 2 -form $\mathbf{u}$. For the moments with support on $T$ we have $\boldsymbol{\alpha} \in \mathcal{I}(4, r-1)$ and

$$
\int_{T} \operatorname{div} \mathbf{u} \lambda^{\boldsymbol{\alpha}}=\int_{\partial T} \mathbf{u} \cdot \mathbf{n}_{T} \lambda^{\boldsymbol{\alpha}}-\int_{T} \mathbf{u} \cdot \operatorname{grad} \lambda^{\boldsymbol{\alpha}}
$$

being $\mathbf{n}_{T}$ the outward pointing normal to $\partial T$.

Example 1: For $r=2, \boldsymbol{\alpha} \in\{(1,0,0,0),(0,1,0,0),(0,0,1,0),(0,0,0,1)\}$. Let $\boldsymbol{\alpha}=(1,0,0,0)$, then $\lambda^{\boldsymbol{\alpha}}=\lambda_{0}$ and $\operatorname{grad} \lambda_{0}=-\sum_{i=1,3} \operatorname{grad} \lambda_{i}$. Remarking that $\mathbf{n}_{T}$ is in agreement (+) or not (-) with that defined on each face according to its own orientation, we get

$$
\begin{aligned}
& \int_{T} \operatorname{div} \mathbf{u} \lambda_{0}=-\int_{\{012\}} \mathbf{u} \cdot \mathbf{n}_{f} \lambda_{0}+\int_{\{013\}} \mathbf{u} \cdot \mathbf{n}_{f} \lambda_{0}-\int_{\{023\}} \mathbf{u} \cdot \mathbf{n}_{f} \lambda_{0} \\
& \quad+\int_{\{123\}} \mathbf{u} \cdot \mathbf{n}_{f} 0-\left(-\int_{T} \mathbf{u} \cdot \operatorname{grad} \lambda_{1}-\int_{T} \mathbf{u} \cdot \operatorname{grad} \lambda_{2}-\int_{T} \mathbf{u} \cdot \operatorname{grad} \lambda_{3}\right) .
\end{aligned}
$$

This explains the first line of the matrix below: the first 4 blocks of 3 entries are associated to the faces of $T$, with no more than one non-zero entry in the first position (that of $\lambda_{0}$ ) and the last block of 3 entries associated to $T$ all equal to 1 because in the volume integrals on the right-hand side there are grad $\lambda_{i}$ with $i=1,2,3$. Same reasoning for the other 3 choices of $\boldsymbol{\alpha}$ which yield the other 3 lines of the matrix below.

$$
\begin{gathered}
{[0,1,2,3]\left[\begin{array}{rrrrrrrrrrrrrrr}
-1 & 0 & 0 & 1 & 0 & 0 & -1 & 0 & 0 & 0 & 0 & 0 & 1 & 1 & 1 \\
0 & -1 & 0 & 0 & 1 & 0 & 0 & 0 & 0 & 1 & 0 & 0 & -1 & 0 & 0 \\
0 & 0 & -1 & 0 & 0 & 0 & 0 & -1 & 0 & 0 & 1 & 0 & 0 & -1 & 0 \\
0 & 0 & 0 & 0 & 0 & 1 & 0 & 0 & -1 & 0 & 0 & 1 & 0 & 0 & -1
\end{array}\right]} \\
{[0,1,2]} \\
{[0,1,3]}
\end{gathered}
$$


(ii) The discrete relation between moments of the 2-form curl $\mathbf{u}$ and those of the 1-form $\mathbf{u}$. In this case, we have to consider two different types of moments, those with support on the faces $f \in \partial T$ and those with support on $T$. For moments with support on a face $f$, we have $\boldsymbol{\alpha} \in \mathcal{I}(3, r-1)$. We denote by $\boldsymbol{\nu}_{f}$ the in-out normal unit vector to $\partial f$ lying on the plane containing the face $f$, and $\boldsymbol{\tau}_{f}=\mathbf{n}_{f} \times \boldsymbol{\nu}_{f}$.

$$
\begin{aligned}
\int_{f} \operatorname{curl} \mathbf{u} \cdot \mathbf{n}_{f} \lambda^{\boldsymbol{\alpha}}= & \int_{f} \operatorname{div}_{f}\left(\mathbf{u} \times \mathbf{n}_{f}\right) \lambda^{\boldsymbol{\alpha}} \\
= & \int_{\partial f}\left(\mathbf{u} \times \mathbf{n}_{f}\right) \cdot \boldsymbol{\nu}_{f} \lambda^{\boldsymbol{\alpha}}-\int_{f} \mathbf{u} \times \mathbf{n}_{f} \cdot \operatorname{grad} \lambda^{\boldsymbol{\alpha}} \\
& =\int_{\partial f} \mathbf{u} \cdot\left(\mathbf{n}_{f} \times \boldsymbol{\nu}_{f}\right) \lambda^{\boldsymbol{\alpha}}-\int_{f} \mathbf{u} \times \mathbf{n}_{f} \cdot \operatorname{grad} \lambda^{\boldsymbol{\alpha}} \\
& =\int_{\partial f} \mathbf{u} \cdot \boldsymbol{\tau}_{f} \lambda^{\boldsymbol{\alpha}}-\int_{f} \mathbf{u} \times \mathbf{n}_{f} \cdot \operatorname{grad} \lambda^{\boldsymbol{\alpha}} .
\end{aligned}
$$

For moments with support on $T, i=\sigma_{T}^{0}(l), l=1,2,3$ and $\boldsymbol{\alpha} \in \mathcal{I}(4, r-2)$.

$$
\begin{aligned}
& \int_{T} \operatorname{curl} \mathbf{u} \cdot \lambda^{\boldsymbol{\alpha}} \operatorname{grad} \lambda_{i} \\
& \quad=-\int_{\partial T} \mathbf{u} \times \mathbf{n}_{T} \cdot \lambda^{\boldsymbol{\alpha}} \operatorname{grad} \lambda_{i}+\int_{T} \mathbf{u} \cdot \operatorname{curl}\left(\lambda^{\boldsymbol{\alpha}} \operatorname{grad} \lambda_{i}\right) \\
& \quad=-\int_{\partial T} \mathbf{u} \times \mathbf{n}_{T} \cdot \lambda^{\boldsymbol{\alpha}} \operatorname{grad} \lambda_{i}+\int_{T} \mathbf{u} \cdot \operatorname{grad} \lambda^{\boldsymbol{\alpha}} \times \operatorname{grad} \lambda_{i} .
\end{aligned}
$$

Example 2: For $r=2$, the face-to-edge and face-to-face blocks in the matrix below are similar (with $f, e$ acting as $T, f$ ) to the corresponding blocks in the matrix of the divergence operator. For the moments on $T$, in this case $\boldsymbol{\alpha}=\mathbf{0}$; the first line of the volume-to-face block results from

$$
\begin{aligned}
& \int_{T} \operatorname{curl} \mathbf{u} \cdot \operatorname{grad} \lambda_{1}=-\left(-\int_{\{012\}} \mathbf{u} \times \mathbf{n}_{f} \cdot \operatorname{grad} \lambda_{1}+\int_{\{013\}} \mathbf{u} \times \mathbf{n}_{f} \cdot \operatorname{grad} \lambda_{1}\right. \\
& \left.-\int_{\{023\}} \mathbf{u} \times \mathbf{n}_{f} \cdot \mathbf{0}+\int_{\{123\}} \mathbf{u} \times \mathbf{n}_{f} \cdot\left[-\operatorname{grad} \lambda_{2}-\operatorname{grad} \lambda_{3}\right]\right)+\int_{T} \mathbf{u} \cdot \mathbf{0} \times \operatorname{grad} \lambda_{1} \\
& {[0,1,2]\left[\begin{array}{rrrrrrrr}
1 & 0 & -1 & 0 & 0 & 0 & 1 & 1 \\
0 & 1 & 0 & 0 & 1 & 0 & -1 & 0 \\
0 & 0 & 0 & -1 & 0 & 1 & 0 & -1
\end{array}\right.} \\
& {[0,1,3] \quad \begin{array}{rlrlllrr}
1 & 0 & -1 & 0 & 0 & 0 & 1 & 1 \\
0 & 1 & 0 & 0 & 1 & 0 & -1 & 0
\end{array}}
\end{aligned}
$$

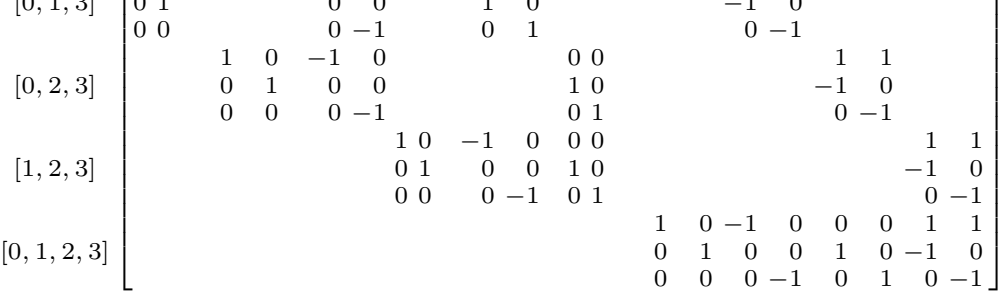

$$
\begin{aligned}
& {[0,1] \quad[0,2] \quad[0,3][1,2] \quad[1,3] \quad[2,3] \quad[0,1,2][0,1,3][0,2,3][1,2,3]}
\end{aligned}
$$

(iii) The discrete relation between moments of the 1-form $\operatorname{grad} u$ and those of the 0 -form $u$. In this case, we have to consider three different types of moments, those with support on the edges $e \in \partial f$ for the faces $f \in \partial T$, those with support on the faces $f \in \partial T$ and those with support on $T$. For moments with support on the edge $e=\left\{n_{i} n_{j}\right\}$, we have $\boldsymbol{\alpha}=\mathcal{I}(2, r-1)$.

$$
\int_{e} \operatorname{grad} u \cdot \boldsymbol{\tau}_{e} \lambda^{\boldsymbol{\alpha}}=\left(u \lambda^{\boldsymbol{\alpha}}\right)\left(n_{j}\right)-\left(u \lambda^{\boldsymbol{\alpha}}\right)\left(n_{i}\right)-\int_{e} u \operatorname{grad} \lambda^{\boldsymbol{\alpha}} \cdot \boldsymbol{\tau}_{e} .
$$


For moments with support on the face $f$, we have $i=\sigma_{f}(l), l=1,2$, and $\boldsymbol{\alpha}=\mathcal{I}(3, r-2)$.

$$
\begin{aligned}
& \int_{f} \operatorname{grad} u \times \mathbf{n}_{f} \cdot \lambda^{\boldsymbol{\alpha}} \operatorname{grad} \lambda_{i}=-\int_{f} \operatorname{grad} u \cdot\left(\lambda^{\boldsymbol{\alpha}} \operatorname{grad} \lambda_{i} \times \mathbf{n}_{f}\right) \\
& \quad=-\left(\int_{\partial f} u\left(\lambda^{\boldsymbol{\alpha}} \operatorname{grad} \lambda_{i} \times \mathbf{n}_{f}\right) \cdot \boldsymbol{\nu}_{f}-\int_{f} u \operatorname{div}_{f}\left(\lambda^{\boldsymbol{\alpha}} \operatorname{grad} \lambda_{i} \times \mathbf{n}_{f}\right)\right) \\
& \quad=-\int_{\partial f} u\left(\lambda^{\boldsymbol{\alpha}} \operatorname{grad} \lambda_{i}\right) \cdot \boldsymbol{\tau}_{f}+\int_{f} u \operatorname{curl}\left(\lambda^{\boldsymbol{\alpha}} \operatorname{grad} \lambda_{i}\right) \cdot \mathbf{n}_{f} \\
& \quad=-\int_{\partial f} u\left(\lambda^{\boldsymbol{\alpha}} \operatorname{grad} \lambda_{i}\right) \cdot \boldsymbol{\tau}_{f}+\int_{f} u\left(\operatorname{grad} \lambda^{\boldsymbol{\alpha}} \times \operatorname{grad} \lambda_{i}\right) \cdot \mathbf{n}_{f} .
\end{aligned}
$$

For the moments with support on $T$, we have $(i, j)=\sigma_{T}^{0}(l, k)$ with $l, k \in$ $\{1,2,3\}, l<k$, and $\boldsymbol{\alpha}=\mathcal{I}(4, r-3)$.

$$
\begin{aligned}
& \int_{T} \operatorname{grad} u \cdot \lambda^{\boldsymbol{\alpha}}\left(\operatorname{grad} \lambda_{i} \times \operatorname{grad} \lambda_{j}\right) \\
& \quad=\int_{\partial T} u \lambda^{\boldsymbol{\alpha}}\left(\operatorname{grad} \lambda_{i} \times \operatorname{grad} \lambda_{j}\right) \cdot \mathbf{n}_{T}-\int_{T} u \operatorname{div}\left(\lambda^{\boldsymbol{\alpha}} \operatorname{grad} \lambda_{i} \times \operatorname{grad} \lambda_{j}\right) \\
& \quad=\int_{\partial T} u \lambda^{\boldsymbol{\alpha}}\left(\operatorname{grad} \lambda_{i} \times \operatorname{grad} \lambda_{j}\right) \cdot \mathbf{n}_{T}-\int_{T} u\left(\operatorname{grad} \lambda^{\boldsymbol{\alpha}} \times \operatorname{grad} \lambda_{i}\right) \cdot \operatorname{grad} \lambda_{j} .
\end{aligned}
$$

Example 3: The matrix below corresponds again to the case $r=2$. So $\operatorname{grad} u$ has not moments with support on $T$, since $r-3<0$. In the face-to-edge block, $\boldsymbol{\alpha}=\mathbf{0}$ and the first line, for instance, follows from

$$
\begin{aligned}
\int_{\{012\}} \operatorname{grad} u \times \mathbf{n}_{f} \cdot \operatorname{grad} \lambda_{1}=-\left(\int_{\{01\}} u \operatorname{grad} \lambda_{1} \cdot \boldsymbol{\tau}_{e}-\int_{\{02\}} u 0\right. \\
\left.+\int_{\{12\}} u\left(-\operatorname{grad} \lambda_{2}\right) \cdot \boldsymbol{\tau}_{e}\right)+\int_{\{012\}} u\left(\mathbf{0} \times \operatorname{grad} \lambda_{1}\right) \cdot \mathbf{n}_{f} .
\end{aligned}
$$

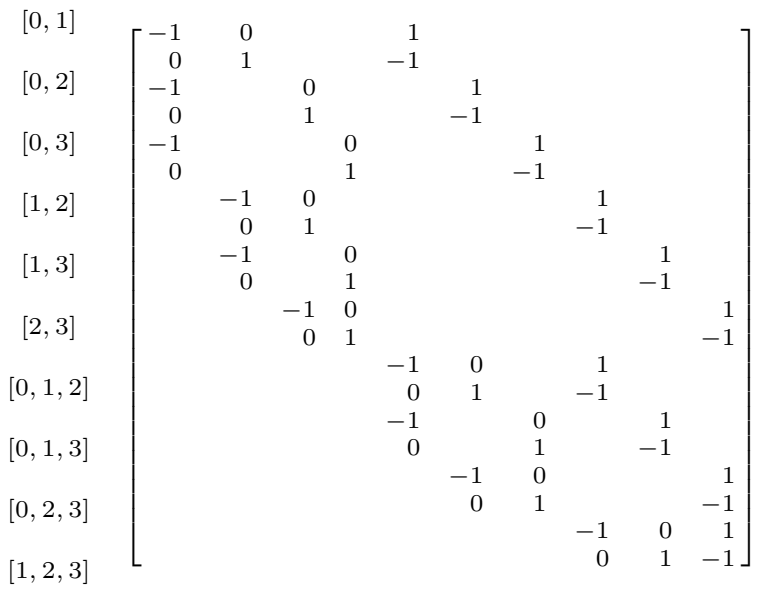

$[0] \quad[1] \quad[2][3] \quad[0,1][0,2][0,3][1,2][1,3][2,3]$

It worths noting that, with a slightly different basis of $\mathcal{P}_{r^{\prime}} \Lambda^{k}(S)$ for $r^{\prime}>2$, the entries of these matrices remain $0,+1$ or -1 .

\section{$3 \quad$ Weights and potentials}

The notation $\{\boldsymbol{\alpha}, S\}$, firstly defined in [7], stands for a particular $k$-simplex, the so-called small $k$-simplices, contained in the (big) tetrahedra $T$ of the 

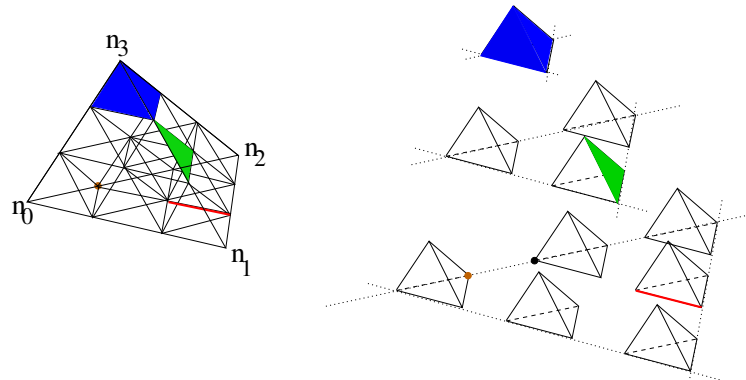

Fig. 1. Visualization (on the left) of all the small simplices associated to the principal lattice of degree $r+1=3$ in the tetrahedron $T=\left\{n_{0} n_{1} n_{2} n_{3}\right\}$. The same set of small simplices in a fragmented visualization (on the right). A small tetrahedron in blue, a small face in green, a small edge in red, and a small nodes in brown (in black, the "twin" node of the brown one in the fragmented view).

mesh. Here $\boldsymbol{\alpha} \in \mathcal{I}(4, r)$ and $S \in \Delta_{k}(T)$. The principal lattice $L_{r+1}(T)$ of order $r+1>1$ in the simplex $T=\left\{n_{\sigma_{T}^{0}(0)} n_{\sigma_{T}^{0}(1)} n_{\sigma_{T}^{0}(2)} n_{\sigma_{T}^{0}(3)}\right\}$ is the set

$L_{r+1}(T)=\left\{\mathbf{x} \in T: \lambda_{\sigma_{T}^{0}(i)}(\mathbf{x}) \in\left\{0, \frac{1}{r+1}, \frac{2}{r+1}, \ldots, \frac{r}{r+1}, 1\right\}, 0 \leq i \leq 3\right\}$.

Let us consider the principal lattice $L_{r+1}(T)$, the multi-index $\boldsymbol{\alpha} \in \mathcal{I}(4, r)$, and a $k$-subsimplex $S$ of $T$. We denote by $\mathbf{v}_{i}$ the (Cartesian) coordinates of the node $n_{i}$ in $\mathbb{R}^{3}$. The small simplex $\{\boldsymbol{\alpha}, S\}$ is the $k$-simplex that belongs to the small tetrahedron with barycenter at the point of coordinates $\sum_{i=0}^{3}\left[\left(\frac{1}{4}+\alpha_{i}\right) \mathbf{v}_{\sigma_{T}^{0}(i)}\right] /(r+1)$, which is parallel and $1 /(r+1)$-homothetic to the (big) sub-simplex $S$ of $T$. For $r+1=3$, in Fig.1, the blue volume is the small tetrahedron $\{(0,0,0,2), T\}$, the green triangle corresponds to the small face $\left\{(0,1,0,1),\left\{n_{1} n_{2} n_{3}\right\}\right\}$, the red segment indicates the small edge $\left\{(0,1,1,0),\left\{n_{0} n_{1}\right\}\right\}$ and all the nodes of $L_{3}(T)$ are small nodes.

Definition 2. The weights of a polynomial $k$-form $u \in \mathcal{P}_{r+1}^{-} \Lambda^{k}(T)$, with $0 \leq k \leq 3$ and $r \geq 0$, are the scalar quantities

$$
\int_{\{\boldsymbol{\alpha}, S\}} u
$$

on the small simplices $\{\boldsymbol{\alpha}, S\}$ with $\boldsymbol{\alpha} \in \mathcal{I}(4, r)$ and $S \in \Delta_{k}(T)$.

The weights (3) of a Whitney $k$-form $u \in \mathcal{P}_{r+1}^{-} \Lambda^{k}(T)$ along all the small simplex $\{\boldsymbol{\alpha}, S\}$ of a mesh $\mathcal{T}$ are unisolvent, as stated in [5, Proposition 3.14]. Since the result on unisolvence holds true also by replacing $T$ with $F \in$ $\Delta_{n-1}(T)$ then $\operatorname{Tr}_{F} u \in \mathcal{P}_{r+1}^{-} \Lambda^{k}(F)$ is uniquely determined by the weights on small simplices in $F$. It thus follows that a locally defined $u$, with $u_{\mid T} \in$ $\mathcal{P}_{r+1}^{-} \Lambda^{k}(T)$ and single-valued weights, is in $H \Lambda^{k}(\Omega)$. We thus can use the weights on the small simplices $\{\boldsymbol{\alpha}, S\}$ as dofs for the fields in the finite element space $\mathcal{P}_{r+1}^{-} \Lambda^{k}(\mathcal{T})$ being aware that their number is greater than the 
dimension of the space. These dofs have again a meaning as cochains and this relates directly the matrix describing the exterior derivative with an incidence matrix. The key point is the Stokes' theorem $\int_{C} \mathrm{~d} u=\int_{\partial C} u$, where $u$ is a $(k-1)$-form and $C$ a $k$-chain. More precisely, if $u \in \mathcal{P}_{r+1}^{-} \Lambda^{k}(\mathcal{T})$ then $z=\mathrm{d} u \in \mathcal{P}_{r+1}^{-} \Lambda^{k+1}(\mathcal{T})$ and

$$
\int_{\{\boldsymbol{\alpha}, S\}} z=\int_{\{\boldsymbol{\alpha}, S\}} \mathrm{d} u=\int_{\partial\{\boldsymbol{\alpha}, S\}} u=\sum_{\{\boldsymbol{\beta}, F\}} B_{\{\boldsymbol{\alpha}, S\},\{\boldsymbol{\beta}, F\}} \int_{\{\boldsymbol{\beta}, F\}} u
$$

being $B$ the incidence matrix with as many rows as small simplices of dimension $k$ and as many columns as small simplices of dimension $k-1$.

The small simplices $\{\boldsymbol{\alpha}, S\}$ inherit the orientation of the simplex $S$ so the coefficient $B_{\{\boldsymbol{\alpha}, S\},\{\boldsymbol{\beta}, F\}}$ is equal to the coefficient $B_{S, F}$ of the incidence of face $F$ on simplex $S$ if $\boldsymbol{\beta}=\boldsymbol{\alpha}$ and zero otherwise. This is straightforward if $\operatorname{dim}(F)>0$ but also when $\operatorname{dim}(F)=0$, as illustrated in Fig.1 (right). The brown dot (on the left) denotes the small node with barycentric coordinates $(2,0,1,0)$ in $T$. In the fragmented visualization, this small node in the notation $\{\boldsymbol{\alpha}, n\}$ becomes $\left\{(2,0,0,0), n_{2}\right\}$ (the brown dot) in $\{(2,0,0,0), T\}$ and $\left\{(1,0,1,0), n_{0}\right\}$ (the black dot) in $\{(1,0,1,0), T\}$.

\section{References}

1. D.N. ARnold, Spaces of finite element differential forms, in Analysis and numerics of partial differential equations, Springer INdAM Ser. 4 (2013) 117-140.

2. D.N. Arnold, R.S. FAlk, And R. Winther, Finite element exterior calculus, homological techniques, and applications, Acta Numer. 15 (2006) 1-155.

3. A. Bossavit, Generating Whitney forms of polynomial degree one and higher IEEE Trans. Magn. 38 (2002) 341-344.

,Bo88 A. Bossavit, Magnetostatic problems in multiply connected regions: some properties of the curl operator, Phys. Sci., Measurement and Instrumentation, Management and Education - Reviews, IEE Procs. A, 135 (1988) 179-187.

4. A. Bossavit, Computational electromagnetism, Academic Press, Inc., San Diego, CA, 1998.

5. S.H. Christiansen And F. Rapetti, On high order finite element spaces of differential forms, Math. Comp. 85 (2016) 517-548.

6. J.-C. NÉDÉLEC, Mixed finite elements in $\mathbf{R}^{3}$, Numer. Math. 35 (1980) 315-341.

7. F. Rapetti And A. Bossavit, Geometrical localization of the degrees of freedom for Whitney elements of higher order, Issue on "Computational Electromagnetism", IEE Proc. Science, Measurement and Technology 1 (2007) 63-66.

8. F. RApetti And A. Bossavit, Whitney forms of higher degree, SIAM J. Numer. Anal. 47 (2009) 2369-2386.

9. P.-A. Raviart, And J.M. Thomas, A mixed finite element method for 2nd order elliptic problems, Mathematical aspects of finite element methods (Proc. Conf., Consiglio Naz. delle Ricerche (C.N.R.), Rome, 1975), Lecture Notes in Math. 606 (1977) 292-315. 
10. H. Whitney, Geometric integration theory, Princeton University Press, Princeton, N. J., 1957. 